\title{
CNU Term Type
}

National Cancer Institute

\section{Source}

National Cancer Institute. CNU Term Type. NCI Thesaurus. Code C54639.

The NCl term type designation for an ISO 3166-1 or Geopolitical Entities, Names, and Codes (GENC) standard country code in numeric (three-digit) format. 